PESQUIMAT, Revista de la Fac.CC.MM. de la

UNIVERSIDAD NACIONAL MAYOR DE SAN MARCOS

Vol.IV, $N^{\circ} 1$,pag.13-29,LIMA-PERÚ. Julio 2001

\title{
CONJUGACIÓN TOPOLÓGICA DE DIFEOMORFISMOS
}

\author{
Martha Gonzáles Bohórquez ${ }^{1}$
}

\begin{abstract}
En el presente trabajo se estudia la conjugación topológica local de un difeomorfismo en espacio de Banach, con su parte lineal en la vecindad de un punto fijo hiperbólico. Para ello se usan herramientas del Análisis Funcional, Aplicaciones de Lipschitz y Teoría Espectral.
\end{abstract}

\section{INTRODUCCIÓN}

Sea $U \subseteq \mathbb{R}^{2}$ un abierto que contiene a $0 \in \mathbb{R}^{n}$ y $f: U \rightarrow \mathbb{R}^{n}$ un difeomorfismo tal que:

1. $f(0)=0$, es decir 0 es punto fijo de $f$.

2. Los autovalores de $D f(0)=L \in G L\left(\mathbb{R}^{n}\right)$, no tienen norma 1, esto es, $L$ es hiperbólico.

En estas condiciones existe $V \subseteq U$ abierto con $0 \in V$ y existe $h: V \rightarrow h[V] \subseteq \mathbb{R}^{n}$ un homeomorfismo tal que $L \circ h=h \circ f$ sobre $V$; es decir, $f$ es localmente topológicamente conjugado con su parte lineal L. Este resultado es conocido como el Teorema de Grobman-Hartman y su demostración puede ser encontrada en cualquier libro de Sistemas Dinámicos. En el presente trabajo se extiende este resultado a espacios vectoriales normados de dimensión infinita (Espacios de Banach) utilizando las técnicas del Análisis Funcional y de los espacios mtricos.

Sea $(E,\|\cdot\|)$ un espacio de Banach y $T \in G L(E)$, esto es $T: E \rightarrow E$ es una aplicación lineal continua biyectiva cuya inversa $T^{-1}$ es también lineal y continua; se dice que:

$$
T \text { es hiperbólico } \Longleftrightarrow \sigma(T) \cap S^{1}=\emptyset \text {. }
$$

donde $\sigma(T)$ es el espectro de $T$ y $S^{1}=\{\lambda \in \mathbb{C}:|\lambda|=1\}$.

\footnotetext{
${ }^{1}$ Universidad Nacional Mayor de San Marcos. Facultad de Ciencias Matemáticas.
} 
En lo que sigue $\operatorname{Hip}(E)$ denotará el espacio de los operadores hiperbólicos en $(E,\|\cdot\|)$. Un resultado de la teoría espectral de operadores lineales es el Teorema de la Descomposición Espectral, el cual, al ser aplicado a los operadores lineales hiperbólicos definidos sobre espacios de Banach nos da como resultado el siguiente:

Teorema 1.1. Sea $(E,\|\cdot\|)$ un espacio de Banach y $L \in \operatorname{Hip}(E)$ entonces existen dos subespacios cerrados $E_{u}$ y $E_{s}$ de $E$; normas $\|\cdot\|_{u}$, $\|\cdot\|_{s} y\|\cdot\|^{*}$ en $E_{u}, E_{s}$ y $E$ respectivamente $y$ una constante positiva a $a \in(0,1)$ que satisfacen:

1. $E=E_{u} \oplus E_{s}$.

2. $L_{u}=\left.L\right|_{E_{u}} \in L\left(E_{u}\right) y\left\|L_{u}^{-1} x_{u}\right\|_{u} \leq a\left\|x_{u}\right\|_{u}, \forall x_{u} \in E_{u} L_{s}=$ $\left.L\right|_{E_{s}} \in L\left(E_{u}\right)$ y $\left\|L_{s} x_{s}\right\|_{s} \leq a\left\|x_{s}\right\|_{s}, \forall x_{s} \in E_{s}$.

3. $\|x\|=\max \left\{\left\|x_{u}\right\|_{u},\left\|x_{s}\right\|_{s}\right\}, x=x_{u}+x_{s}, x_{u} \in E_{u}, x_{s} \in E_{s}$ donde la norma $\|\cdot\|^{*}$ es equivalente a la norma inicial $\|\cdot\|$ de $E$.

Las funciones Lipschitzianas con constantes de Lipschitz menor que 1 , son llamadas contracciones. Las contracciones son de gran importancia cuando están definidas en un espacio métrico completo, ya que en este caso, ellas poseen un único punto fijo atractor.

Son de gran utilidad en la demostración del Teorema de GrobmanHartman los siguientes teoremas:

Teorema 1.2. Sea $(X, d)$ un espacio métrico completo y $f: X \rightarrow X$ Lipschitziana con constante de Lipschitz Lip $(f)<1$. Entonces existe un único $x_{0} \in X$ tal que:

1. $f\left(x_{0}\right)=x_{0}\left(x_{0}\right.$ es un punto fijo de $\left.f\right)$.

2. $\lim _{n \rightarrow \infty} f^{n}(x)=x_{0}, \forall x \in X$ ( $x_{0}$ es atractor).

Teorema 1.3.(De la función inversa de Lipschitz) Sea $(E,\|\cdot\|)$ un espacio de Banach, $\varphi: E \rightarrow E$ Lipschitziana y $L \in G L(E)$ tal que $\operatorname{Lip}(\varphi)<\left\|L^{-1}\right\|^{-1}$, entonces $L+\varphi$ es inversible, $(L+\varphi)^{-1}$ es Lipschitziana $y$

$$
\operatorname{Lip}\left[(L+\varphi)^{-1}\right] \leq \frac{1}{\left\|L^{-1}\right\|^{-1}-\operatorname{Lip}(\varphi)}
$$

Previo a la demostración del Teorema de Grobman-Hartman en espacios de Banach se probarán dos Lemas que son de aplicación directa en la demostración de dicho teorema. 


\section{PRELIMINARES}

Definición 2.1. Sea $(E,\|\cdot\|)$ un espacio de Banach, $U$ una vecindad de $0 \in E$ y $f: U \rightarrow E$ un difeomorfismo sobre su imagen, 0 es punto fijo hiperbólico de $f$ si:

1. $f(0)=0$.

2. $D f(0) \in H i p(E)$.

Es importante recordar que si $\left(E,\|\cdot\|_{E}\right),\left(F,\|\cdot\|_{F}\right)$ son dos espacios de Banach entonces el espacio de las funciones continuas y acotadas de $E$ a $F$ denotado por $C_{b}(E, F)$ es también un espacio de Banach.

Si $E=F$, en lugar de $C_{b}(E, E)$ se escribirá $C_{b}(E)$.

En $E$ consideramos la norma $\|\cdot\|^{*}$ que satisface (3) del Teorema 1.1. y que denotaremos con $\|\cdot\|$, esto es $\|\cdot\|^{*}=\|\cdot\|$.

El Teorema 1.1., nos dice que todo operador lineal hiperbólico descompone al espacio de Banach $E$ donde está definido en suma directa de dos subespacios cerrados de $E$, invariantes por el operador.

Probaremos que la descomposición de $E$ en suma directa de $E_{u}$ y $E_{s}$ induce una descomposición de $C_{b}(E)$ en suma directa de $C_{b}\left(E, E_{u}\right)$ y $C_{b}\left(E, E_{s}\right)$.

Empecemos definiendo las proyecciones canónicas $\pi_{u}$ y $\pi_{s}$

$$
\begin{array}{rlrl}
\pi_{u}: E & \rightarrow E_{u} & \pi_{s}: E & \rightarrow E_{s} \\
x & \mapsto \pi_{u}(x)=x_{u} & x & \mapsto \pi_{s}(x)=x_{s}
\end{array}
$$

donde $x=x_{u}+x_{s} \in E_{u} \oplus E_{s}=E$.

Es inmediato ver la linealidad de $\pi_{u} \mathrm{y} \pi_{s}$.

Como

$$
\left\|\pi_{u}(x)\right\|_{u}=\left\|x_{u}\right\|_{u} \leq \max \left\{\left\|x_{u}\right\|_{u},\left\|x_{s}\right\|_{s}\right\}=\|x\|, \quad \forall x \in E
$$

y

$$
\left\|\pi_{s}(x)\right\|_{s}=\left\|x_{s}\right\|_{s} \leq \max \left\{\left\|x_{u}\right\|_{u},\left\|x_{s}\right\|_{s}\right\}=\|x\|, \quad \forall x \in E
$$

se sigue que $\pi_{u}$ y $\pi_{s}$ son operadores lineales y acotados, luego son continuos es decir $\pi_{u} \in L\left(E, E_{u}\right)$ y $\pi_{s} \in L\left(E, E_{s}\right)$. Además $\left\|\pi_{u}\right\| \leq 1$ y $\left\|\pi_{s}\right\| \leq 1$.

Sea $f \in C_{b}(E)$, definimos $f_{u}=\pi_{u} \circ f$ y $f_{s}=\pi_{s} \circ f$, es decir 


$$
\begin{array}{rlrl}
f_{u}: E & \rightarrow E_{u} & f_{s}: & E \\
x & \mapsto f_{u}(x)=\pi_{u}(f(x)) & & \rightarrow E_{s} \\
& & \mapsto f_{s}(x)=\pi_{s}(f(x))
\end{array}
$$

como $\pi_{u} \in L\left(E, E_{u}\right) \subseteq C_{b}\left(E, E_{u}\right)$ y $\pi_{s} \in L\left(E, E_{s}\right) \subseteq C_{b}\left(E, E_{s}\right)$ entonces $f_{u} \in C_{b}\left(E, E_{u}\right)$ y $f_{s} \in C_{b}\left(E, E_{s}\right)$.

Mostraremos que: $f=f_{u}+f_{s}$

$x \in E \Longrightarrow f(x) \in E=E_{u} \oplus E_{s} \Longrightarrow f(x)=[f(x)]_{u}+[f(x)]_{s} \in E_{u}+E_{s}$ luego

$\left(\pi_{u} \circ f\right)(x)=\pi_{u}(f(x))=[f(x)]_{u} \quad$ y $\quad\left(\pi_{s} \circ f\right)(x)=\pi_{s}(f(x))=[f(x)]_{s}$

esto es: $f_{u}(x)=[f(x)]_{u}$ y $f_{s}(x)=[f(x)]_{s}$, entonces $f(x)=f_{u}(x)+$ $f_{s}(x), \forall x \in E$; es decir $f=f_{u}+f_{s} \in C_{b}\left(E, E_{u}\right)+C_{b}\left(E, E_{s}\right)$. Por tanto

$$
C_{b}(E)=C_{b}\left(E, E_{u}\right)+C_{b}\left(E, E_{s}\right)
$$

Ahora tomemos $f \in C_{b}\left(E, E_{u}\right) \cap C_{b}\left(E, E_{s}\right)$ esto es $f \in C_{b}\left(E, E_{u}\right)$ y $f \in C_{b}\left(E, E_{s}\right)$; entonces para $x \in E$ se tiene $f(x) \in E_{u} \cap E_{s}$ y como $E_{u} \cap E_{s}=\{0\}$ entonces $f(x)=0, \forall x \in E$, es decir $f=0$. Así

$$
C_{b}\left(E, E_{u}\right) \cap C_{b}\left(E, E_{s}\right)=\{0\}
$$

De (2.1) y (2.2) queda probado que

$$
C_{b}(E)=C_{b}\left(E, E_{u}\right) \oplus C_{b}\left(E, E_{s}\right)
$$

Teniendo en cuenta que $(E,\|\cdot\|)$ es un espacio de Banach y que los subespacios normados $E_{u}$ y $E_{s}$ con normas $\|\cdot\|_{u}$ y $\|\cdot\|_{s}$ respectivamente, son subespacios cerrados de $E$, resulta inmediato ver que $\left(E_{u},\|\cdot\|_{u}\right)$ y $\left(E_{s},\|\cdot\|_{s}\right)$ son espacios de Banach. Por consiguiente, es claro también que

$$
\left(C_{b}\left(E, E_{u}\right),\|\cdot\|_{E, E_{u}}\right), \quad\left(C_{b}\left(E, E_{s}\right),\|\cdot\|_{E, E_{s}}\right) \quad \text { y } \quad\left(C_{b}(E),\|\cdot\|_{E, E}\right)
$$

son espacios de Banach.

La relación existente entre las normas $\|\cdot\|_{E, E_{u}},\|\cdot\|_{E, E_{s}} \mathrm{y}\|\cdot\|_{E, E}$ está dada en la siguiente afirmación:

$$
\|f\|_{E, E}=\max \left\{\left\|f_{u}\right\|_{E, E_{u}},\left\|f_{s}\right\|_{E, E_{s}}\right\}, \quad \forall f \in C_{b}(E)
$$


En efecto, para $x \in E$, se tiene $f(x)=f_{u}(x)+f_{s}(x) \in E=E_{u}+E_{s}$. Como $\|f(x)\|=\max \left\{\left\|f_{u}(x)\right\|_{u},\left\|f_{s}(x)\right\|_{s}\right\}$, tenemos $\left\|f_{u}(x)\right\|_{u} \leq$ $\|f(x)\|$ y $\left\|f_{s}(x)\right\|_{s} \leq\|f(x)\|, \forall x \in E$. Luego

$$
\sup _{x \in E}\left\|f_{u}(x)\right\|_{u} \leq \sup _{x \in E}\|f(x)\| \quad \text { y } \quad \sup _{x \in E}\left\|f_{s}(x)\right\|_{s} \leq \sup _{x \in E}\|f(x)\|
$$

entonces $\left\|f_{u}\right\|_{E, E_{u}} \leq\|f\|_{E, E} \quad$ y $\quad\left\|f_{s}\right\|_{E, E_{s}} \leq\|f\|_{E, E}$. Concluimos que

$$
\max \left\{\left\|f_{u}\right\|_{E, E_{u}},\left\|f_{s}\right\|_{E, E_{s}}\right\} \leq\|f\|_{E, E}
$$

Por otro lado, se tiene

$\left\|f_{u}(x)\right\|_{u} \leq\left\|f_{u}\right\|_{E, E_{u}} \leq \max \left\{\left\|f_{u}\right\|_{E, E_{u}},\left\|f_{s}\right\|_{E, E_{s}}\right\}, \quad \forall x \in E$

$\left\|f_{s}(x)\right\|_{s} \leq\left\|f_{s}\right\|_{E, E_{s}} \leq \max \left\{\left\|f_{u}\right\|_{E, E_{u}},\left\|f_{s}\right\|_{E, E_{s}}\right\}, \quad \forall x \in E$ luego

$$
\max \left\{\left\|f_{u}(x)\right\|_{u},\left\|f_{s}(x)\right\|_{s}\right\} \leq \max \left\{\left\|f_{u}\right\|_{E, E_{u}},\left\|f_{s}\right\|_{E, E_{s}}\right\}
$$

es decir $\|f(x)\| \leq \max \left\{\left\|f_{u}\right\|_{E, E_{u}},\left\|f_{s}\right\|_{E, E_{s}}\right\}, \forall x \in E$ entonces

$$
\sup _{x \in E}\|f(x)\| \leq \max \left\{\left\|f_{u}\right\|_{E, E_{u}},\left\|f_{s}\right\|_{E, E_{s}}\right\}
$$

Por lo tanto

$$
\|f\|_{E, E} \leq \max \left\{\left\|f_{u}\right\|_{E, E_{u}},\left\|f_{s}\right\|_{E, E_{s}}\right\}
$$

De (2.3) y (2.4) queda probado que

$$
\|f\|_{E, E}=\max \left\{\left\|f_{u}\right\|_{E, E_{u}},\left\|f_{s}\right\|_{E, E_{s}}\right\} .
$$

Ahora consideremos $T \in L\left(C_{b}(E)\right)$ tal que

$$
T_{u}=\left.T\right|_{C_{b}\left(E, E_{u}\right)} \in L\left(C_{b}\left(E, E_{u}\right)\right) \quad \text { y } \quad T_{s}=\left.T\right|_{C_{b}\left(E, E_{s}\right)} \in L\left(C_{b}\left(E, E_{s}\right)\right) \text {. }
$$

Para $f \in C_{b}(E)$ se tiene que $T(f) \in C_{b}(E)$, luego

$$
f=f_{u}+f_{s} \quad \text { y } \quad T(f)=[T(f)]_{u}+[T(f)]_{s}
$$

donde $f_{u},[T(f)]_{u} \in C_{b}\left(E, E_{u}\right)$ y $f_{s},[T(f)]_{s} \in C_{b}\left(E, E_{s}\right)$. Pero: 


$$
\begin{aligned}
{[T(f)]_{u} } & =\pi_{u}(T(f))=\pi_{u}\left(T\left(f_{u}+f_{s}\right)\right)=\pi_{u}\left(T\left(f_{u}\right)+T\left(f_{s}\right)\right) \\
& =\pi_{u}\left(T_{u}\left(f_{u}\right)+T_{s}\left(f_{s}\right)\right)=T_{u}\left(f_{u}\right)
\end{aligned}
$$

y

$$
\begin{aligned}
{[T(f)]_{s} } & =\pi_{s}(T(f))=\pi_{s}\left(T\left(f_{u}+f_{s}\right)\right)=\pi_{s}\left(T\left(f_{u}\right)+T\left(f_{s}\right)\right) \\
& =\pi_{s}\left(T_{u}\left(f_{u}\right)+T_{s}\left(f_{s}\right)\right)=T_{s}\left(f_{s}\right)
\end{aligned}
$$

entonces

$$
\|T(f)\|_{E, E}=\max \left\{\left\|T_{u}\left(f_{u}\right)\right\|_{E, E_{u}},\left\|T_{s}\left(f_{s}\right)\right\|_{E, E_{s}}\right\}
$$

Probaremos que: $\|T\|=\max \left\{\left\|T_{u}\right\|,\left\|T_{s}\right\|\right\}, \forall T \in L\left(C_{b}(E)\right)$.

En efecto,

$$
\begin{aligned}
\left\|T_{u}\left(f_{u}\right)\right\|_{E, E_{u}} & \leq\left\|T_{u}\right\|\left\|f_{u}\right\|_{E, E_{u}} \leq\left\|T_{u}\right\|\|f\|_{E, E} \\
& \leq \max \left\{\left\|T_{u}\right\|,\left\|T_{s}\right\|\right\}\|f\|_{E, E}
\end{aligned}
$$

también:

$$
\begin{aligned}
\left\|T_{s}\left(f_{s}\right)\right\|_{E, E_{s}} & \leq\left\|T_{s}\right\|\left\|f_{s}\right\|_{E, E_{s}} \leq\left\|T_{s}\right\|\|f\|_{E, E} \\
& \leq \max \left\{\left\|T_{u}\right\|,\left\|T_{s}\right\|\right\}\|f\|_{E, E}
\end{aligned}
$$

luego

$\max \left\{\left\|T_{u}\left(f_{u}\right)\right\|_{E, E_{u}},\left\|T_{s}\left(f_{s}\right)\right\|_{E, E_{s}}\right\} \leq \max \left\{\left\|T_{u}\right\|,\left\|T_{s}\right\|\right\}\|f\|_{E, E}$ es decir:

$\|T(f)\|_{E, E} \leq \max \left\{\left\|T_{u}\right\|,\left\|T_{s}\right\|\right\}\|f\|_{E, E}, \quad \forall f \in C_{b}(E)$.

Por lo tanto

$$
\|T\|=\max \left\{\left\|T_{u}\right\|,\left\|T_{s}\right\|\right\} .
$$

Recíprocamente:

$$
\begin{aligned}
\left\|T_{u}\left(f_{u}\right)\right\|_{E, E_{u}} & =\left\|T\left(f_{u}\right)\right\|_{E, E_{u}}=\left\|T\left(f_{u}\right)\right\|_{E, E} \\
& \leq\|T\|\left\|f_{u}\right\|_{E, E_{u}}, \quad \forall f_{u} \in C_{b}\left(E, E_{u}\right)
\end{aligned}
$$

entonces $\left\|T_{u}\right\| \leq\|T\|$.

Además 


$$
\begin{aligned}
\left\|T_{s}\left(f_{s}\right)\right\|_{E, E_{s}} & =\left\|T\left(f_{s}\right)\right\|_{E, E_{s}}=\left\|T\left(f_{s}\right)\right\|_{E, E} \\
& \leq\|T\|\left\|f_{s}\right\|_{E, E_{s}}, \quad \forall f_{s} \in C_{b}\left(E, E_{s}\right)
\end{aligned}
$$

entonces $\left\|T_{s}\right\| \leq\|T\|$

Por lo tanto:

$$
\max \left\{\left\|T_{u}\right\|,\left\|T_{s}\right\|\right\} \leq\|T\| .
$$

De (2.6) y (2.7) está probado que $\|T\|=\max \left\{\left\|T_{u}\right\|,\left\|T_{s}\right\|\right\}$.

\section{DOS LEMAS PREVIOS}

El Teorema de Grobman-Hartman establece que "Sea $(E,\|\cdot\|)$ un espacio de Banach, $U$ un abierto de $E$ tal que $0 \in U, f: U \rightarrow E$ difeomorfismo de clase $C^{1}$ sobre su imagen y sea 0 punto fijo hiperbólico de $f$. Denotemos $L=D f(0) \in H i p(E)$. Entonces $f$ es localmente conjugado a $L$, es decir existe $h \in H$ Hom $(E)$ tal que $L \circ h=h \circ f$ en una vecindad del 0 contenida en U". Para demostrarlo, necesitamos de los dos siguientes Lemas que probaremos en seguida.

Lema 3.1. Sea $(E,\|\cdot\|)$ un espacio de Banach, $U$ un abierto de $E$ tal que $0 \in U$, sea $f: U \rightarrow E$ de clase $c^{1}$ sobre su imagen tal que $f(0)=0$ $y$ denotemos por $L=D f(0) \in L(E)$. Entonces $\forall \epsilon>0, \exists r=r(\epsilon)>0$ y $\exists \varphi \in C_{b}(E) \cap \operatorname{Lip}(E)$ tal que $f=L+\varphi$ en $B_{r}(0) \subseteq U$.

Demostración. Consideremos $\phi: U \rightarrow E$ definida por $\phi(x)=f(x)-$ $L(x)$, entonces $\phi \in C^{1}$, puesto que $f \in C^{1}$ y $D \phi(x)=D f(x)-L$. Además:

$$
\begin{aligned}
\phi(0) & =f(0)-L(0)=0-0=0 \\
D \phi(0) & =D f(0)-L=L-L=0
\end{aligned}
$$

Procuramos extender $\phi$ a todo $E$ de modo tal que su extensión sea continua, acotada y Lipschitziana con constante de Lipschitz menor que $\epsilon$. Para ello, consideremos $\alpha: \mathbb{R} \rightarrow[0,1]$ una funcin de clase $C^{\infty}$ que satisface:

$$
\alpha(t)= \begin{cases}1, & \text { si }|t| \leq 1 / 2 \\ 0, & \text { si }|t| \geq 1\end{cases}
$$

Como $D \phi$ es continua en 0 , dado $\epsilon>0$, existe $\delta=\delta(\epsilon)>0$ tal que 


$$
\|x\|<\delta \Longrightarrow\|D \phi(x)\|<\frac{\epsilon}{k+1}
$$

en donde $\delta>0$ es tomado suficientemente pequeño tal que $\overline{B_{\delta}(0)} \subseteq U$

Definamos $\varphi: E \rightarrow E$ tal que

$$
\varphi(x)= \begin{cases}\alpha(\|x\| / \delta), & \text { si } x \in U \\ 0, & \text { si } x \in E-U\end{cases}
$$

Es claro que $\varphi$ es continua en $U$, y desde que para $x \in U$ con $\|x\|>\delta$, $\varphi(x)=\alpha(\|x\| / \delta) \phi(x)=0$ entonces $\varphi$ es continua en $E$. Probemos que es acotada en $E$. Ya que $\varphi$ se anula fuera de la bola $B_{\delta}(0)$, será suficiente probar que $\varphi$ es acotada en $B_{\delta}(0)$.

Sea $x \in B_{\delta}(0)$, se tiene:

$$
\begin{aligned}
\|\varphi(x)\| & =\|\alpha(\|x\| / \delta) \phi(x)\|=|\alpha(\|x\| / \delta)|\|\phi(x)\| \\
& \leq\|\phi(x)\|=\|\phi(x)-\phi(0)\| \leq\left(\sup _{x \in B_{\delta}(0)}\|D \phi(x)\|\right)\|x\| \\
& \leq \frac{\epsilon}{K+1} \delta .
\end{aligned}
$$

Por lo tanto

$$
\|\varphi(x)\| \leq \frac{\epsilon}{K+1} \delta, \quad \forall x \in B_{\delta}(0)
$$

De esta forma se tiene probado que $\varphi$ es continua y acotada en $E$, es decir $\varphi \in C_{b}(E)$. Ahora probaremos que $\varphi$ es Lipschitziana. Para $x_{1}, x_{2} \in U$, se tiene:

$$
\begin{aligned}
\left\|\varphi\left(x_{1}\right)-\varphi\left(x_{2}\right)\right\|= & \left\|\alpha\left(\left\|x_{1}\right\| / \delta\right) \phi\left(x_{1}\right)-\alpha\left(\left\|x_{2}\right\| / \delta\right) \phi\left(x_{2}\right)\right\| \\
\leq & \left\|\alpha\left(\left\|x_{1}\right\| / \delta\right) \phi\left(x_{1}\right)-\alpha\left(\left\|x_{2}\right\| / \delta\right) \phi\left(x_{1}\right)\right\| \\
& +\left\|\alpha\left(\left\|x_{2}\right\| / \delta\right) \phi\left(x_{1}\right)-\alpha\left(\left\|x_{2}\right\| / \delta\right) \phi\left(x_{2}\right)\right\|
\end{aligned}
$$

Entonces:

$$
\begin{aligned}
(3.1)\left\|\varphi\left(x_{1}\right)-\varphi\left(x_{2}\right)\right\| \leq & \alpha\left(\frac{\left\|x_{1}\right\|}{\delta}\right)-\alpha\left(\frac{\left\|x_{2}\right\|}{\delta}\right) \mid\left\|\phi\left(x_{1}\right)\right\|+ \\
& \alpha\left(\frac{\left\|x_{2}\right\|}{\delta}\right) \mid\left\|\phi\left(x_{1}\right)-\phi\left(x_{2}\right)\right\|
\end{aligned}
$$


Sean $x_{1}, x_{2} \in E$. Pongámonos en el caso que $x_{1}, x_{2} \in B_{\delta}(0)$. Sabemos, por el Teorema del Valor Medio, que existe $t^{*}$ entre $\frac{\left\|x_{1}\right\|}{\delta}$ y $\frac{\left\|x_{2}\right\|}{\delta}$ tal que:

$$
\begin{aligned}
\left|\alpha\left(\frac{\left\|x_{1}\right\|}{\delta}\right)-\alpha\left(\frac{\left\|x_{2}\right\|}{\delta}\right)\right| & \leq\left|\alpha\left(t^{*}\right)\right| \mid \frac{\left\|x_{1}\right\|}{\delta}-\frac{\left\|x_{2}\right\|}{\delta} \\
& \leq \frac{K\left\|x_{1}-x_{2}\right\|}{\delta} .
\end{aligned}
$$

Usando la desigualdad de Lagrange se tiene:

$$
\begin{aligned}
\left\|\phi\left(x_{1}\right)\right\| & =\left\|\phi\left(x_{1}\right)-\phi(0)\right\| \leq\left(\sup _{x \in B_{\delta}(0)}\|D \phi(x)\|\right)\left\|x_{1}\right\| \\
\leq & \frac{\epsilon}{K+1} \delta \\
\left\|\phi\left(x_{1}\right)-\phi\left(x_{2}\right)\right\| & \leq\left(\sup _{x \in B_{\delta}(0)}\|D \phi(x)\|\right)\left\|x_{1}-x_{2}\right\| \\
& \leq \frac{\epsilon}{K+1}\left\|x_{1}-x_{2}\right\|
\end{aligned}
$$

Reemplazando (3.2), (3.3) y (3.4) en (3.1) se tiene que

$$
\begin{aligned}
\left\|\varphi\left(x_{1}\right)-\varphi\left(x_{2}\right)\right\| & \leq \frac{K}{\delta}\left\|x_{1}-x_{2}\right\| \frac{\epsilon}{K+1} \delta+\frac{\epsilon}{K+1}\left\|x_{1}-x_{2}\right\| \\
& =\epsilon\left\|x_{1}-x_{2}\right\|
\end{aligned}
$$

Supongamos ahora que $x_{1} \in B_{\delta}(0)$ y $x_{2} \notin B_{\delta}(0)$. Si $x_{2} \in U$, ya que $\alpha\left(\frac{\left\|x_{2}\right\|}{\delta}\right)=0$, de (3.1) tenemos:

$$
\left\|\varphi\left(x_{1}\right)-\varphi\left(x_{2}\right)\right\| \leq\left|\alpha\left(\frac{\left\|x_{1}\right\|}{\delta}\right)-\alpha\left(\frac{\left\|x_{2}\right\|}{\delta}\right)\right|\left\|\phi\left(x_{1}\right)\right\|
$$

Reemplazando (3.2) y (3.3) en (3.5)

$$
\begin{aligned}
\left\|\varphi\left(x_{1}\right)-\varphi\left(x_{2}\right)\right\| & \leq \frac{K}{\delta}\left\|x_{1}-x_{2}\right\| \frac{\epsilon}{K+1} \delta=\frac{K}{K+1} \epsilon\left\|x_{1}-x_{2}\right\| \\
& <\epsilon\left\|x_{1}-x_{2}\right\|
\end{aligned}
$$

Ahora asumimos que $x_{1} \in B_{\delta}(0)$ y $x_{2} \notin B_{\delta}(0)$, con $x_{2} \notin U$. Entonces 


$$
\begin{aligned}
\left\|\varphi\left(x_{1}\right)-\varphi\left(x_{2}\right)\right\| & =\left\|\varphi\left(x_{1}\right)\right\|=\left\|\alpha\left(\frac{\left\|x_{1}\right\|}{\delta}\right) \phi\left(x_{1}\right)\right\| \\
& =\alpha\left(\frac{\left\|x_{1}\right\|}{\delta}\right)-\alpha\left(\frac{\left\|x_{2}\right\|}{\delta}\right) \mid\left\|\phi\left(x_{1}\right)\right\|
\end{aligned}
$$

Luego, de (3.2) y (3.3) tenemos:

$$
\begin{aligned}
\left\|\varphi\left(x_{1}\right)-\varphi\left(x_{2}\right)\right\| & \leq \frac{K}{\delta}\left\|x_{1}-x_{2}\right\| \frac{\epsilon}{K+1} \delta=\frac{K}{K+1} \epsilon\left\|x_{1}-x_{2}\right\| \\
& <\epsilon\left\|x_{1}-x_{2}\right\|
\end{aligned}
$$

Por último, en el caso que $x_{1}, x_{2} \notin B_{\delta}(0)$ se tiene:

$$
\left\|\varphi\left(x_{1}\right)-\varphi\left(x_{2}\right)\right\|=0<\epsilon\left\|x_{1}-x_{2}\right\| .
$$

En cualquier caso $\left\|\varphi\left(x_{1}\right)-\varphi\left(x_{2}\right)\right\| \leq \epsilon\left\|x_{1}-x_{2}\right\|$, entonces $\varphi \epsilon$ $\operatorname{Lip}(E)$ y $\operatorname{Lip}(\varphi)<\epsilon$. Hasta ahora tenemos $\varphi \in C_{b}(E) \cap \operatorname{Lip}(E)$ con $\operatorname{Lip}(\varphi)<\epsilon$. Finalmente veamos que en una vecindad del 0 contenida en $U$.

$$
\|x\| \leq \frac{\delta}{2} \Longrightarrow \frac{\|x\|}{\delta} \leq \frac{1}{2} \Longrightarrow \alpha\left(\frac{\|x\|}{\delta}\right)=1
$$

luego $\varphi(x)=\phi(x)=f(x)-L(x)$ entonces $f(x)=L(x)+\varphi(x)$, tomando $r=\frac{\delta}{2}$ tenemos $f=L+\varphi$ en $B_{r}(0) \subset U$.

Lema 3.2. Sea $(E,\|\cdot\|)$ un espacio de Banach, y $L \in H i p(E)$, si $\epsilon>0$ es suficientemente pequeño, entonces $\forall \varphi, \psi \in \operatorname{Lip}(E) \cap C_{b}(E)$ con $\operatorname{Lip}(\varphi), \operatorname{Lip}(\psi)<\epsilon$, la ecuación funcional:

$$
(L+\varphi) \circ(I+w)=(I+w) \circ(L+\psi)
$$

tiene una única solución en $C_{b}(E)$.

Demostración. Tomamos la ecuación funcional dada y establecemos algunas equivalencias:

$$
\begin{aligned}
& (L+\varphi) \circ(I+w)=(I+w) \circ(L+\psi) \Longleftrightarrow \\
& L+L \circ w+\varphi \circ(I+w)=L+\psi+w \circ(L+\psi) \Longleftrightarrow \\
& L \circ w-w \circ(L+\psi)=\psi-\varphi \circ(I+w) \Longleftrightarrow \\
& w-L^{-1} \circ w \circ(L+\psi)=L^{-1} \circ(\psi-\varphi \circ(I+w)) \Longleftrightarrow \\
& (I-L) w=L^{-1} \circ(\psi-\varphi \circ(I+w))
\end{aligned}
$$


donde $L(w)=L^{-1} \circ w \circ(L+\psi)$.

Ahora procuramos resolver ésta última ecuación equivalente. Observe que si $(I-L)$ fuera inversible, se tendría $w=(I-L)^{-1} \circ L^{-1} \circ$ $(\psi-\varphi \circ(I+w))$ y haciendo $T(w)=(I-L)^{-1} \circ L^{-1} \circ(\psi-\varphi \circ(I+w))$ se tiene $T(w)=w$. De ser $T$ una contracción, ella tendría un único punto fijo, el cual sería la única solución de la ecuación funcional dada. Entonces todo se reduce a probar que:

1. $I-L$ es inversible.

2. $T$ es una contracción.

(1) Probaremos que $(I-L)$ es inversible:

Para $w \in C_{b}(E), L(w)=L^{-1} \circ w \circ(L+\psi)$ con $\psi \in C_{b}(E) \cap \operatorname{Lip}(E)$ y $L \in H i p(E)$. Ya que $L \in H i p(E)$, entonces $L, L^{-1} \in L(E)$, luego:

$$
\psi \in C_{b}(E) \Longrightarrow L+\psi \in C_{b}(E) \Longrightarrow L^{-1} \circ w \circ(L+\psi) \in C_{b}(E)
$$

es decir

$$
\begin{aligned}
L: C_{b}(E) & \rightarrow \mathbb{C}_{b}(E) \\
w & \mapsto L(w)=L^{-1} \circ w \circ(L+\psi)
\end{aligned}
$$

Observe que $L$ es lineal. En efecto, sean $C_{1}, C_{2} \in \mathbb{R}, w_{1}, w_{2} \in C_{b}(E)$, se cumple:

$$
\begin{aligned}
L\left(C_{1} w_{1}+C_{2} w_{2}\right) & =L^{-1} \circ\left(C_{1} w_{1}+C_{2} w_{2}\right) \circ(L+\psi) \\
& =L^{-1} \circ\left[\left(C_{1} w_{1}\right) \circ(L+\psi)+\left(C_{2} w_{2}\right) \circ(L+\psi)\right] \\
& =C_{1} L^{-1} \circ w_{1} \circ(L+\psi)+C_{2} L^{-1} \circ w_{2} \circ(L+\psi) \\
& =C_{1} L\left(w_{1}\right)+C_{2} L\left(w_{2}\right)
\end{aligned}
$$

Por lo tanto $L \in L\left(C_{b}(E)\right)$

Además

$$
\begin{aligned}
w \in C_{b}\left(E, E_{u}\right) & \Longrightarrow w \circ(L+\psi) \in C_{b}\left(E, E_{u}\right) \\
& \Longrightarrow L^{-1} \circ w \circ(L+\psi) \in C_{b}\left(E, E_{u}\right) \\
w \in C_{b}\left(E, E_{s}\right) & \Longrightarrow w \circ(L+\psi) \in C_{b}\left(E, E_{s}\right) \\
& \Longrightarrow L^{-1} \circ w \circ(L+\psi) \in C_{b}\left(E, E_{s}\right)
\end{aligned}
$$

Es decir

$$
L_{u}=\left.L\right|_{C_{b}\left(E, E_{u}\right)} \in L\left(C_{b}\left(E, E_{u}\right)\right) \quad \text { y } \quad L_{s}=\left.L\right|_{C_{b}\left(E, E_{s}\right)} \in L\left(C_{b}\left(E, E_{s}\right)\right)
$$


Afirmación 1: $I_{u}-L_{u}$ es inversible:

En efecto, Sea $w \in C_{b}\left(E, E_{u}\right)$ y $x \in E$ entonces $w \circ(L+\psi)(x) \in E_{u}$ desde que $L \in \operatorname{Hip}(E)$, por el Teorema 1.1 se sigue que $\exists a, 0<a<1$. que satisface:

$$
\begin{gathered}
\left\|L^{-1} \circ w^{\prime} \circ(L+\psi)(x)\right\|_{u} \leq a\|w \circ(L+\psi)(x)\|_{u} \leq a\|w\|_{E, E_{u}} \\
\left\|L_{u}(w)(x)\right\|_{u} \leq a\|w\|_{E, E_{u}}, \quad \forall x \in E \\
\left\|L_{u}(w)\right\|_{E, E_{u}}=\sup _{x \in E}\left\|L_{u}(w)(x)\right\|_{u} \leq a\|w\|_{E, E_{u}}, \quad \forall x w \in C_{b}\left(E, E_{u}\right)
\end{gathered}
$$

Por lo tanto

$$
\left\|L_{u}\right\| \leq a<1
$$

Ya que $L_{u} \in L\left(C_{b}\left(E, E_{u}\right)\right)$ con $\left\|L_{u}\right\|<1$, entonces

$$
I_{u}-L_{u} \in G L\left(C_{b}\left(E, E_{u}\right)\right)
$$

y además

$$
\left\|\left(I_{u}-L_{u}\right)^{-1}\right\| \leq \frac{1}{1-\left\|L_{u}\right\|} \leq \frac{1}{1-a}
$$

Afirmación 2: $I_{s}-L_{s}$ es inversible:

Para probar esta afirmación empleamos otro método, ya que con el anterior usado en la afirmación 1 , se presenta el inconveniente de no contar con una forma conocida de acotar $L_{s}^{-1}$. Sabemos que $(E,\|\cdot\|)$ es de Banach, $L \in G L(E)$ y $\psi \in \operatorname{Lip}(E)$, si se cumpliera que $\operatorname{Lip}(\psi) \leq$ $\left\|L^{-1}\right\|^{-1}$ entonces por Teorema $1.3, L+\psi$ sería inversible, $(L+\psi)^{-1} \bar{\epsilon}$ $\operatorname{Lip}(E)$ y

$$
\operatorname{Lip}\left((L+\psi)^{-1}\right) \leq \frac{1}{\left\|L^{-1}\right\|^{-1}-\operatorname{Lip}(\psi)},
$$

lo que estaría garantizando la buena definición de:

$$
\begin{aligned}
L_{s}^{*}: C_{b}\left(E, E_{s}\right) & \rightarrow \mathbb{C}_{b}\left(E, E_{s}\right) \\
w & \mapsto L_{s}^{*}(w)=L_{s} \circ w \circ(L+\psi)^{-1}
\end{aligned}
$$

Resulta inmediato verificar que $L_{s} \circ L_{s}^{*}=I_{s}$ y $L_{s}^{*} \circ L_{s}=I_{s}$, por consiguiente $L_{s}^{*}=L_{s}^{-1}$. Como $L_{s}$ es inversible y $I_{s}-L_{s}=L_{s}\left(L_{s}^{-1}-I_{s}\right)$, $I_{s}-L_{s}$ será inversible; si $L_{s}^{-1}-I_{s}$ es inversible. Probemos que $L_{s}^{-1}-I_{s}$ es inversible. Sea $w \in C_{b}\left(E, E_{s}\right)$ y $x \in E$ entonces $w \circ(L+\psi)^{-1}(x) \in E_{s}$ luego, por el Teorema 1.1 se tiene: 


$$
\begin{aligned}
\left\|L_{s}^{-1}(w)(x)\right\|_{s} & =\left\|L_{s} \circ w \circ(L+\psi)^{-1}(x)\right\|_{s} \\
& \leq a\left\|w \circ(L+\psi)^{-1}(x)\right\|_{s} \leq a\|w\|_{E, E_{s}}, \quad \forall x \in E \\
\left\|L_{s}^{-1}(w)\right\|_{E, E_{s}}= & \sup _{x \in E}\left\|L_{s}^{-1}(w)(x)\right\|_{s} \leq a\|w\|_{E, E_{s}}, \quad \forall w \in C_{b}\left(E, E_{s}\right)
\end{aligned}
$$

Por lo tanto

$$
\left\|L_{s}^{-1}\right\| \leq a<1
$$

Ya que $L_{s}^{-1} \in L\left(C_{b}\left(E, E_{s}\right)\right)$ con $\left\|L_{s}^{-1}\right\|<1$, entonces

$$
I_{s}-L_{s}^{-1} \in G L\left(C_{b}\left(E, E_{u}\right)\right)
$$

es decir $I_{s}-L_{s}^{-1}$ es inversible y

$$
\left(I_{s}-L_{s}^{-1}\right)^{-1} \in L\left(C_{b}\left(E, E_{s}\right)\right) \subset \operatorname{Lip}\left(C_{b}\left(E, E_{s}\right)\right)
$$

y además

$$
\operatorname{Lip}\left(\left(I_{s}-L_{s}^{-1}\right)^{-1}\right)=\left\|\left(I_{s}-L_{s}^{-1}\right)^{-1}\right\| \leq \frac{1}{1-\left\|L_{s}^{-1}\right\|} \leq \frac{1}{1-a}
$$

Luego $I_{s}-L_{s}=L_{s}\left(L_{s}^{-1}-I_{s}\right)$ es inversible y $\left(I_{s}-L_{s}\right)^{-1}=\left(L_{s}^{-1}-\right.$ $\left.I_{s}\right)^{-1} L_{s}^{-1}$, por lo tanto $\left(I_{s}-L_{s}\right)^{-1} \in L\left(C_{b}\left(E, E_{s}\right)\right) \subset \operatorname{Lip}\left(C_{b}\left(E, E_{s}\right)\right)$ y además

$$
\begin{aligned}
\left\|\left(I_{s}-L_{s}\right)^{-1}\right\| & =\operatorname{Lip}\left[\left(I_{s}-L_{s}\right)^{-1}\right] \leq \operatorname{Lip}\left[\left(L_{s}^{-1}-I_{s}\right)^{-1}\right] \operatorname{Lip}\left(L_{s}^{-1}\right) \\
& \leq \frac{\left\|L_{s}^{-1}\right\|}{1-a} \leq \frac{a}{1-a}
\end{aligned}
$$

Por lo tanto:

$$
\left\|\left(I_{s}-L_{s}\right)^{-1}\right\| \leq \frac{a}{1-a}
$$

Afirmación 3: $(I-L)^{-1}=\left(I_{u}-L_{u}\right)^{-1}+\left(I_{s}-L_{s}\right)^{-1}$. En efecto:

$$
\begin{gathered}
(I-L) \circ\left[\left(I_{u}-L_{u}\right)^{-1}+\left(I_{s}-L_{s}\right)^{-1}\right]= \\
=(I-L) \circ\left(I_{u}-L_{u}\right)^{-1}+(I-L) \circ\left(I_{s}-L_{s}\right)^{-1} \\
=\left(I_{u}-L_{u}\right) \circ\left(I_{u}-L_{u}\right)^{-1}+\left(I_{s}-L_{s}\right) \circ\left(I_{s}-L_{s}\right)^{-1}=I_{u}+I_{s}=I
\end{gathered}
$$

y también

$$
\begin{gathered}
{\left[\left(I_{u}-L_{u}\right)^{-1}+\left(I_{s}-L_{s}\right)^{-1}\right] \circ(I-L)=} \\
=\left[\left(I_{u}-L_{u}\right)^{-1}+\left(I_{s}-L_{s}\right)^{-1}\right] \circ\left[\left(I_{u}-L_{u}\right)+\left(I_{s}-L_{s}\right)\right]
\end{gathered}
$$




$$
=\left(I_{u}-L_{u}\right)^{-1} \circ\left(I_{u}-L_{u}\right)+\left(I_{s}-L_{s}\right)^{-1} \circ\left(I_{s}-L_{s}\right)=I_{u}+I_{s}=I
$$

En conclusión, siempre que $\operatorname{Lip}(\psi) \leq\left\|L^{-1}\right\|^{-1}$, se tiene que $I-L$ es inversible, $(I-L)^{-1} \in L\left(C_{b}(E)\right)$ y

$$
\begin{aligned}
\left\|(I-L)^{-1}\right\| & =\max \left\{\left\|\left(I_{u}-L_{u}\right)^{-1}\right\|,\left\|\left(I_{s}-L_{s}\right)^{-1}\right\|\right\} \\
& \leq \max \left\{\frac{1}{1-a}, \frac{a}{1-a}\right\}=\frac{1}{1-a}
\end{aligned}
$$

(2) Probaremos que $T$ es contracción. Para $w \in C_{b}(E)$ definimos $T(w)=(I-L)^{-1} \circ L^{-1} \circ(\psi-\varphi \circ(I+w))$. Observe que en realidad $T=T(\psi, \varphi)$ con $\psi, \varphi \in \operatorname{Lip}(E) \cap C_{b}(E)$. Observemos que:

$$
\varphi \circ(I+w) \in C_{b}(E) \Longrightarrow \psi-\varphi \circ(I+w) \in C_{b}(E)
$$

luego $(I-L)^{-1} \circ L^{-1} \circ(\psi-\varphi \circ(I+w)) \in C_{b}(E)$, es decir $T(w) \in C_{b}(E)$. Así se tiene

$$
\begin{aligned}
T: C_{b}(E) & \rightarrow \mathbb{C}_{b}(E) \\
w & \mapsto T(w)=(I-L)^{-1} \circ L^{-1} \circ(\psi-\varphi \circ(I+w))
\end{aligned}
$$

Dados $w_{1}, w_{2} \in C_{b}(E)$, tenemos:

$$
\begin{aligned}
\| T\left(w_{1}\right)= & T\left(w_{2}\right)\left\|_{E, E}=\right\|(I-L)^{-1} \circ L^{-1} \circ\left(\psi-\varphi \circ\left(I+w_{1}\right)\right)- \\
& -(I-L)^{-1} \circ L^{-1} \circ(\psi-\varphi \circ(I+w)) \|_{E, E} \\
= & \left\|(I-L)^{-1} \circ L^{-1} \circ\left(-\varphi \circ\left(I+w_{1}\right)+\varphi \circ\left(I+w_{2}\right)\right)\right\|_{E, E} \\
\leq & \left.\left\|(I-L)^{-1}\right\| \cdot\left\|L^{-1}\right\| \cdot \| \varphi \circ\left(I+w_{1}\right)+\varphi \circ\left(I+w_{2}\right)\right) \|_{E, E} \\
\leq & \frac{\left\|L^{-1}\right\|}{1-a} \operatorname{Lip}(\varphi)\left\|w_{1}-w_{2}\right\|_{E, E}
\end{aligned}
$$

$T$ será contracción si $T \in \operatorname{Lip}\left(C_{b}(E)\right)$ y $\operatorname{Lip}(T)<1$, para conseguir esto basta tomar $\operatorname{Lip}(\varphi)<(1-a)\left\|L^{-1}\right\|^{-1}$. Desde que $C_{b}(E)$ es un espacio de Banach y $T$ una contracción, por el Teorema 1.2: Existe un único $w_{0} \in C_{b}(E)$ tal que $T\left(w_{0}\right)=w_{0}$, el cual es solución de la ecuación funcional dada. Ya que $\operatorname{Lip}(\psi), \operatorname{Lip}(\varphi)<\epsilon$, es suficiente tomar:

$$
\epsilon \leq \min \left\{\left\|L^{-1}\right\|^{-1},(1-a)\left\|L^{-1}\right\|^{-1}\right\}=(1-a)\left\|L^{-1}\right\|^{-1} .
$$

Corolario 3.3. Sea $(E,\|\cdot\|)$ un espacio de Banach y $L \in \operatorname{Hip}(E)$. Si $0<\epsilon<(1-a)\left\|L^{-1}\right\|^{-1}$, entonces $(L+\varphi)$ y $(L+\psi)$ son conjugados para todo par $\varphi, \psi \in C_{b}(E) \cap \operatorname{Lip}(E)$ tales que $\operatorname{Lip}(\psi), \operatorname{Lip}(\varphi)<\epsilon$. 
Demostración. Por el Lema anterior, la ecuación funcional:

$$
(L+\varphi) \circ(I+w)=(I+w) \circ(L+\psi)
$$

tiene solución única $w_{0} \in C_{b}(E)$. Análogamente la ecuación funcional:

$$
(L+\psi) \circ(I+\tilde{w})=(I+\tilde{w}) \circ(L+\varphi)
$$

tiene solución única $\tilde{w}_{0} \in C_{b}(E)$. De (3.6) y (3.7):

$$
\begin{aligned}
& \left(I+w_{0}\right)\left(I+\tilde{w}_{0}\right)(L+\varphi)=\left(I+w_{0}\right)(L+\psi)\left(I+\tilde{w}_{0}\right)=(L+\varphi)\left(I+w_{0}\right)\left(I+\tilde{w}_{0}\right) \\
& \left(I+\tilde{w}_{0}\right)\left(I+w_{0}\right)(L+\varphi)=\left(I+\tilde{w}_{0}\right)(L+\psi)\left(I+w_{0}\right)=(L+\varphi)\left(I+\tilde{w}_{0}\right)\left(I+w_{0}\right)
\end{aligned}
$$

donde las ecuaciones:

$$
\begin{aligned}
& (I+\tilde{w})(I+\tilde{w})(L+\varphi)=(L+\varphi)\left(I+w_{0}\right)\left(I+\tilde{w}_{0}\right) \\
& (I+\tilde{w})(I+w)(L+\psi)=(L+\psi)(I+\tilde{w})(I+w)
\end{aligned}
$$

tienen solución única del tipo $I+w$ con $w \in C_{b}(E)$ puesto que

$(I+w)(I+\tilde{w})=I+(\tilde{w}+w+w \tilde{w}),(I+\tilde{w})(I+w)=I+(w+\tilde{w}+\tilde{w} w)$ y $\tilde{w}+w+w \tilde{w}, w+\tilde{w}+\tilde{w} w \in C_{b}(E)$, desde que $w, \tilde{w} \in C_{b}(E)$.

Es obvio que $(I+0)(L+\varphi)=(L+\varphi)(I+0)$ y $(I+0)(L+\psi)=$ $(L+\psi)(I+0)$ luego por la unicidad de la solución del tipo $I+w$, con $w \in C_{b}(E)$; de las ecuaciones funcionales (3.8) se tiene:

$$
\left(I+w_{0}\right)\left(I+\tilde{w}_{0}\right)=I=\left(I+\tilde{w}_{0}\right)\left(I+w_{0}\right)
$$

luego $\left(I+w_{0}\right)^{-1}=\left(I+\tilde{w}_{0}\right) \in C_{b}(E)$, luego haciendo $h=I+w_{0}$, se tiene que $h \in H o m(E)$ y de (7.6):

$$
(L+\varphi) \circ h=h \circ(L+\psi)
$$

Por lo tanto $L+\varphi$ y $L+\psi$ son conjugados.

\section{EL TEOREMA DE GROBMAN-HARTMAN PARA DIFEOMORFISMOS EN ESPACIOS DE BANACH}

Estamos ya preparados para demostrar nuestro resultado principal:

Teorema 4.1.((El Teorema de Grobman-Hartman para difeomorfismos en espacio de Banach) Sea $(E,\|\cdot\|)$ un espacio de Banach, $U$ 
un abierto de $E$ tal que $0 \in U, f: U \rightarrow E$ difeomorfismo de clase $C^{1}$ sobre su imagen y sea 0 punto fijo hiperbólico de $f$. Denotemos $L=D f(0) \in \operatorname{Hip}(E)$. Entonces $f$ es localmente conjugado a L. es decir existe $h \in H o m(E)$ tal que $L \circ h=h \circ f$ en $B_{r}(0) \subseteq U$.

Demostración. Sea $\epsilon>0$ tal que $\epsilon<\left.(1-a)\left\|L^{-1}\right\|\right|^{-1}$ y puesto que $f(0)=0$, por el Lema 3.1 existe un $r>0$ y existe un $\varphi \in \operatorname{Lip}(E) \cap$ $C_{b}(E)$ con $\operatorname{Lip}(\varphi)<\epsilon$ tal que $f=L+\varphi$ en $B_{r}(0) \subseteq U$.

Tomemos $\psi=0$, es obvio que $\psi \in \operatorname{Lip}(E) \cap C_{b}(E)$ con $\operatorname{Lip}\left(\iota^{\prime}\right)=$ $0<\epsilon$. Como $\operatorname{Lip}(\varphi), \operatorname{Lip}(\psi)<\epsilon<(1-a)\left\|L^{-1}\right\|^{-1}$. por el Corolario $3.3 L+\varphi$ y $L$ son conjugados; esto es existe $h \in H o m(E)$ tal que

$$
L h=h(L+\varphi)
$$

Pero $f=L+\varphi$ en $B_{r}(0) \subseteq U$ entonces $L h=h f$ en $B_{r}(0) \subseteq U$. Se sigue que $L$ y $f$ son conjugados en $B_{r}(0)$.

Observación: 0 es punto fijo de $h$, es decir $h(0)=0$. En efecto. como $L h=h f$ en $B_{r}(0), L h(0)=h f(0)$ entonces $L(h(0))=h(0)$. $h(0) \in E=E_{u} \oplus E_{s}$, denotemos $h(0)=x_{u}+x_{s} \operatorname{con} x_{u} \in E_{u} \mathrm{y} x_{s} \in E_{s}$

$$
\begin{gathered}
L\left(x_{u}+x_{s}\right)=x_{u}+x_{s} \Longrightarrow L x_{u}+L x_{s}=x_{u}+x_{s} \Longrightarrow L x_{u}=x_{u} \text { y } L \cdot x_{s}=x_{s} \\
\left\|x_{s}\right\|_{s}=\left\|L_{s} x_{s}\right\|_{s} \leq a\left\|x_{s}\right\|_{s} \Longrightarrow(1-a)\left\|x_{s}\right\|_{s} \leq 0 \Longrightarrow\left\|x_{s}\right\|_{s}=0 \\
\Longrightarrow x_{s}=0 \\
\left\|x_{u}\right\|_{u}=\left\|L_{u}^{-1} x_{u}\right\|_{u} \leq a\left\|x_{u}\right\|_{u} \\
\Longrightarrow(1-a)\left\|x_{u}\right\|_{u} \leq 0 \Longrightarrow\left\|x_{u}\right\|_{u}=0
\end{gathered}
$$

Por lo tanto $h(0)=0+0=0$.

\section{BIBLIOGRAFÍA}

[1] Hirch, M. - Smale, S., Differential Equations, Dynamical Systems and Linear Algebra, Academic Press New York 1976.

[2] Hirch, M. - Pugh, W. - Shub, M., Invariant Manifolds, Lectures Notes in Mathematics N 583, Springer - Verlag, New York 1976.

[3] Irwin, M., On the stable manifold theorem, Bull, London Math. Soc. 2 (1970).

[4] Kreyszig, E., Introductory Functional Analysis with Applications, John Wiley and Sons, New York. Chichester. Brisbame. Toronto. Singapore, 1989.

[5] Nachbin, L. , Introdução a Analise Funcional: Espaços de Banach e Cálculo Diferencial, Serie de matemática, monografía N 17., Secretaría Geral da Organizaç ão dos Estados Americanos. Programa Regional de Desenvolvimento Científico e Tecnológico. 1982. 
[6] Nitecki, Z., Differentiable Dynamics, M.I.T. 1971.

[7] Palis J., de Melo, W., Geometric Theory of Dynamical Systems, Springer Verlag 1980 .

[8] Rudin, W., Functional Analysis, Mc Graw-Hill, 1991.

[9] Chain Höning. C., Análise Funcional e Aplicações, Vol. II, Publicaçóes do Instituto de Matemática e Estatística da Universidade de São Paulo, 1970.

[10] Lang, S., Real Analysis, Addison - Wesley Publishing Company.

[11] Lang, S., Analysis I, Addison - Wesley Reading Mass., 1968.

[12] Shub, M., Global Stability of Dynamical Systems, Springer-Verlag, 1990.

[13] Smale, S., Differentiable Dynamical Systems, Bulletin AMS, 1967.

14] Sotomayor, J., Lições de Equações Diferenciais Ordinarias, Projeto Euclides. IMPA, 1979

[15] Dieudonne, J., Fundamentos de Análisis Moderno.Editorial Reverté S.A. 1976. 